\title{
Intersektorale Vernetzung im Gesundheitswesen: Prozessverbesserung durch den Einsatz von Plattformen am Fallbeispiel der Palliativversorgung
}

\author{
Alina Behne $\mathbb{D} \cdot$ Frank Teuteberg
}

Eingegangen: 15. Dezember 2020 / Angenommen: 20. Mai 2021 / Online publiziert: 23. Juni 2021

(C) Der/die Autor(en) 2021

Zusammenfassung Eine effiziente Überführung der Patienten in die häusliche Versorgung steht bei der Palliativentlassung insbesondere aufgrund der häufig kurzen Versorgungsdauer im Fokus. Dabei besteht die Herausforderung, dass viele unterschiedliche Akteure an dem Prozess beteiligt sind. Plattformen bieten Potenziale, eine einfache Dokumentation und Kommunikation der an der Versorgung beteiligten Gesundheitsakteure zu ermöglichen. Der vorliegende Beitrag zeigt, wie das Zusammenspiel unterschiedlicher Akteure auf Prozess- und Personenebene untersucht werden kann, um anschließend Prozessschwachstellen zu erkennen und diese mittels Informations- und Kommunikationstechnologien zu verbessern. In Zusammenarbeit mit verschiedenen Praxisakteuren vor allem aus dem Gesundheitswesen aber auch aus der Softwareentwicklung konnten aktuelle Herausforderungen im Palliativbereich erhoben werden. Daran anknüpfend konnte eine Anforderungsanalyse abgeleitet, ein bestehendes System erweitert und anschließend der Entlass- und Versorgungsprozess restrukturiert werden. Dabei werden Bedarfe, Potenziale und Herausforderungen von der Erhebung des aktuellen Prozesses bis zur Implementierung der Restrukturierung hervorgehoben. Es wird deutlich, dass das intersektorale Medikationsmanagement ein Schlüsselelement in der Gesundheitsversorgung ist und die Apotheke die Rolle eines Gesundheitslotsen einnehmen könnte. Bislang ist noch keine standardisierte oder einheitliche intersektorale Kommunikation oder Dokumentation via Plattform im Gesundheitswesen etabliert, jedoch bestehen einsatzbereite Plattformlösungen, die den Austausch erleichtern und den Weg bis zur Entstehung einer einheitlichen Lösung für alle beteiligten Akteure ebnen. Dieser Beitrag zeigt, wie die Erweiterung einer bestehenden Plattform aus dem Pallia-

\footnotetext{
Alina Behne $(\varangle) \cdot$ Frank Teuteberg

Fachgebiet Unternehmensrechnung und Wirtschaftsinformatik, Universität Osnabrück, Osnabrück, Deutschland

E-Mail: alina.behne@uni-osnabrueck.de
} 
tivsektor es ermöglichen kann, die hier untersuchten Prozesse in Hinblick auf eine sektorenübergreifende, effiziente Zusammenarbeit zu verbessern.

Schlüsselwörter Plattform · Palliativversorgung · Prozessverbesserung · Kommunikation · Gesundheitswesen

\title{
Intersectoral connectivity in healthcare: Process improvement by the utilization of platforms based on a case study in palliative care
}

\begin{abstract}
An efficient transition of the palliative patient from hospital to home care should be focused due to its short duration of care. This faces the challenge of many different actors being involved in the process of palliative care. In this context, platforms offer potential to enable effective documentation and communication between the healthcare actors involved in the care process. This study shows how the interaction of different actors can be investigated at the process and person level in order to subsequently identify process weaknesses and improve them using technologies. In cooperation with various practitioners, especially from the healthcare sector but also from software development, current challenges in the palliative care sector could be identified. Based on this, a requirements analysis was derived, an existing system was enhanced and then the discharge and care process of palliative care was restructured. The study highlights needs and challenges from the elicitation of the current process to the implementation of the restructured process. It becomes clear that intersectoral medication management is a key element in healthcare and that pharmacists could adopt the role of a healthcare manager. To date, no standardized or consistent intersectoral communication or documentation via platform has been established in healthcare, but ready-to-use platform solutions exist to facilitate exchange now and pave the way until a single solution is developed for all. An efficient way to optimize processes is to extend existing platforms. On this basis, this study uses the palliative sector to illustrate how cross-sector, efficient collaboration via these platforms can look in a restructured process.
\end{abstract}

Keywords Platform · Palliative care - Process improvement - Communication · Healthcare

\section{Einleitung}

Der Technologieeinsatz für die Palliativversorgung ist ein kleines, aber sich stark entwickelndes Forschungsfeld (Ostherr et al. 2016). Ziel der Palliativversorgung ist die angemessene Betreuung von Menschen mit einer unheilbaren, fortschreitenden Krankheit an den Wohnorten ihrer letzten Lebensphase. Der Palliativsektor ist - von der Entlassung bis zur häuslichen Versorgung - durch das Zusammenwirken vieler unterschiedlicher Akteure gekennzeichnet. Darüber hinaus ist die ambulante Palliativversorgung im eigenen Zuhause des Patienten aufgrund der meist kurzen Versorgungsdauer und der Notwendigkeit von schnell verfügbaren Betäubungsmitteln hochsensibel, weshalb eine einfache Kommunikation der an der Versorgung 
beteiligten Gesundheitsakteure von besonderer Bedeutung ist. Damit Technologien an richtiger Stelle eingesetzt werden können, bedarf es zuerst einer Darstellung des Zusammenspiels der zahlreichen Akteure für die Palliativversorgung auf Prozessund Personenebene (Mouttham et al. 2012). Im Rahmen der ambulanten Versorgung von Patienten mit fortschreitenden, unheilbaren Erkrankungen kann von den Hausärzten bei schwierigen Symptomen bei Bedarf zusätzlich ein örtliches Palliativnetz involviert werden. Diese mobilen multiprofessionellen Teams bestehen aus Palliativärzten, Palliativpflegekräften sowie weiteren Professionen (z. B. Sozialdienst, Musiktherapie, Physiotherapie). In einigen Regionen Deutschlands sind in diese Palliativnetze auch die Hausärzte integriert. Ein wichtiges Angebot dieser Dienste sind ihre 24-Stunden-Erreichbarkeit und Versorgung der Patienten in ihrer häuslichen Umgebung. Auch die zuständigen Pflegedienste spielen hier eine wichtige Rolle. Neben dem Palliativnetz gibt es noch den Begriff palliativmedizinischer Konsiliardienst (PKD), welcher den Vertragspartner der Krankenversicherung darstellt. Im weiteren Verlauf dieses Artikels werden Palliativnetz und PKD synonym verwendet.

Aktuell bieten digitale Palliativ-Fallakten bzw. -systeme den Palliativärzten und -pflegern mithilfe eines übersichtlichen Medikationsmanagements und der Dokumentation von Arztbefunden die Möglichkeit, einen Palliativpatienten qualitätsgerecht und effizient zu versorgen. Bislang nutzen jedoch überwiegend nur Palliativnetze derartige patientenzentrierte Plattformen. Dabei beginnt der palliative Versorgungprozess eines Patienten vor Einschaltung der spezifischen Leistungserbringer, bereits bei der Entlassung aus der Klinik. Hausärzte für die Einschreibung in ein Palliativnetz und Apotheker für die Medikamentenversorgung übernehmen dann die Versorgung, bevor im letzten Schritt das Palliativnetz hinzugezogen werden kann. Dies ist zu Beginn der Betreuung meist der Herausforderung gegenübergestellt, zahlreiche analog gepflegte oder nicht standardisierte, digital erstellte und anschließend ausgedruckte Dokumente überblicken zu müssen. Das Palliativnetz muss initial also einen hohen Aufwand leisten, adäquate Daten für die Versorgung zu verarbeiten. Ein großes Potenzial einer gemeinsamen patientenzentrierten Plattform ist der Zugriff auf dieselbe Datenbasis. Es wurden bereits mehrere Prozesse von Gesundheitsinstitutionen digitalisiert, jedoch meist nicht vollständig, sodass teilweise wieder auf die analoge Papierform zurückgegriffen wird und eine Hybridform entsteht (Mumm et al. 2021). Darüber hinaus überschreiten diese Digitalisierungsprozesse oftmals nicht die eigenen Unternehmensgrenzen. Die Vernetzung verschiedener Akteure stellt eine große Herausforderung dar, kann aber zu einer Reduzierung der analogen Ablage, zu einer besseren Kommunikation der Akteure und schließlich zu einer Zeitersparnis und zu Kosteneinsparungen führen (Osmani et al. 2008). Es fehlt die sektorenübergreifende Nutzung, um eine qualitativ hochwertige Betreuung von Palliativpatienten effizient zu ermöglichen (Bönisch 2016). Der Palliativbereich wurde in diesem Vorhaben als Problembereich identifiziert, da von der Entlassung des Patienten bis zur häuslichen Versorgung des Palliativpatienten neben dem Palliativnetz auch Klinik, Hausarzt und Apotheker in dem Prozess eingebunden sind.

Ziel des Artikels besteht darin zu zeigen, welche Schwachstellen in dem aktuellen Prozess zur Palliativentlassung vorliegen und wie der Einsatz von Plattformen diesen begegnen kann. Darüber hinaus wird deutlich, wie IT-gestützte Fallakten für Pal- 
liativpatienten auf unterschiedliche Gesundheitsversorger erweitert werden können und wie ein Soll-Prozess unter Einbindung aller Akteure aussieht. Neben der Umsetzung der Integration dieser digital-unterstützten Prozesse liegt ein weiteres Ziel des Artikels darin, Klarheit über die Vorteile des Einsatzes einer bereichsübergreifenden Plattform für die beteiligten Akteure zu schaffen. Innerhalb dieses Vorhabens wird daher die folgende Forschungsfrage verfolgt:

Wie können Prozesse im Gesundheitswesen (im Speziellen im Palliativbereich) durch Kommunikations- und Dokumentationsplattformen effizienter gestaltet werden?

\section{Methodik}

Diese vorliegende Untersuchung umfasst verschiedene Methoden, um Prozesse zu erheben, aktuelle Anforderungen an Plattformen für eine verbesserte intersektorale Vernetzung im Palliativbereich zu konsolidieren und anschließend umsetzen zu können. Als zentrale Methode orientiert sich dieses Vorhaben am Geschäftsprozessmanagement-Lebenszyklus, bei dem sukzessiv und repetitiv in der Gesamtheit die folgenden fünf Schritte durchgeführt werden: Zu Beginn wird der Prozess erhoben, anschließend modelliert, analysiert, verbessert und zuletzt implementiert (Dumas et al. 2013). Das Vorgehen und die verwendeten Methoden werden ausführlich in Abb. 1 dargestellt und beziehen sich auf das operative Prozessmanagement.

Die hohe Komplexität und Mehrdimensionalität von Alltagsproblemen kann unter Verwendung verschiedener Methoden besser erfasst werden (Mingers 2001). Neben einer Literaturrecherche steht bei diesem Ansatz die Expertise von Praktikern im Vordergrund, welche durch Fokusgruppen (Morgan 1997) und Experteninterviews (Gläser and Laudel 2010) aggregiert werden konnte. Unter Verwendung der Prozessmodellierungssprache Business Process Model and Notation (BPMN) konnten erhobene Informationen anschließend in Prozessform konsolidiert modelliert werden. Die BPMN hat sich in den vergangenen Jahren zur effizienten Erstellung von Geschäftsprozessen durch die intuitive und detaillierte Repräsentation des Prozessflows bewährt (Wohed et al. 2006). Nach der Prozesserhebung wird der Prozess analysiert und werden Schwachstellen identifiziert, um diese anschließend in ei-

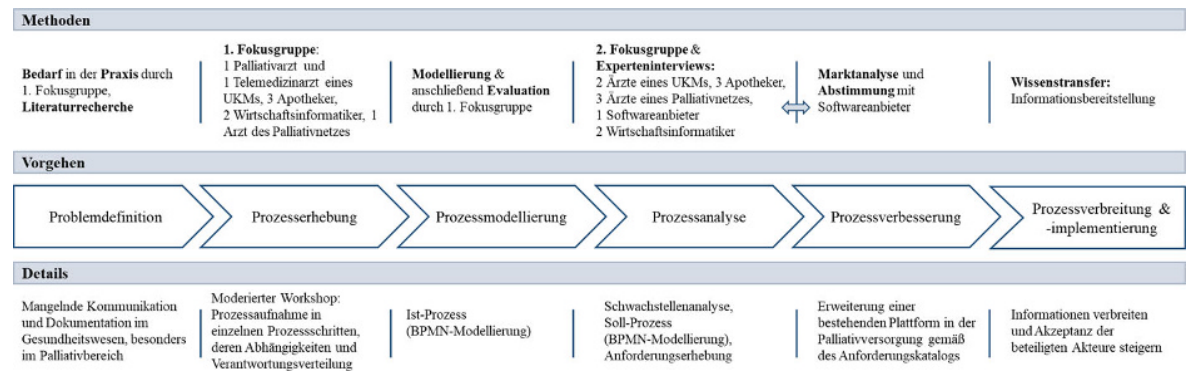

Abb. 1 Zugrundeliegende Methodik dieses Vorhabens (angelehnt an Dumas et al. 2013) 
ner Restrukturierung, u. a. durch den Einsatz digitaler Technologien, zu einem SollProzess beheben zu können (Coskun et al. 2008). Die Informationen in der Prozesserhebung und -analyse basieren zum Großteil auf den Erfahrungswerten der Experten und teils auf Literatur. Das vollständige Prozessverständnis trägt dazu bei, eine Anforderungsanalyse an ein System erstellen zu können, welche anschließend $\mathrm{zu}$ einer besseren Lösung für ein bestehendes soziales und organisationales Problem führt (Mingers 2001).

\section{Potenziale von Plattformen zur intersektoralen Kommunikation und Dokumentation im Palliativsektor}

\subsection{Prozesserhebung, -modellierung und -analyse: Identifikation von Prozessschwachstellen}

Insgesamt konnte im Palliativsektor ein starkes Aufkommen von Kommunikationsschwierigkeiten und redundanter Dokumentenablage identifiziert werden. Zur Aufnahme des Ist-Prozesses in BPMN wurden die an der Entlassung und Versorgung von Palliativpatienten beteiligten Gesundheitsakteure in Bezug zu ihrem Alltagsprozess befragt. Die Aussagen der Akteure wurden anschließend zusammengetragen, sodass ein abstrahierter Ist-Prozess ${ }^{1}$ entstand, der auch auf Palliativnetze in anderen Regionen übertragen werden kann. An dem Entlass- und Versorgungsprozess von Palliativpatienten sind insgesamt sechs unterschiedliche Gruppen beteiligt: Patienten, Kliniken, Angehörige, Hausärzte, Apotheken und Palliativnetze. In dem modellierten Ist-Prozess wurde aufgrund der Übersichtlichkeit die Rolle des Patienten und der Angehörigen zusammengefasst. Für die Definition eines Soll-Prozesses ${ }^{2}$ wurden Herausforderungen des Ist-Prozesses in den Mittelpunkt gestellt, um diesen mithilfe der Möglichkeiten der Digitalisierung zu begegnen. Dieser Soll-Prozess stellt einen kommunikativen, transparenten und effizienten Prozess unter Anwendung einer bereichsübergreifenden Austauschplattform zwischen den Gesundheitsakteuren dar, der bei Umsetzung aller Akteure einen Best Practice-Fall bieten kann.

Im aktuellen Prozess wird die Kommunikation erschwert, da die führenden Informations- und Kommunikationsmedien im Einsatz Telefon und Fax sind. Dabei greifen Gesundheitsdienstleister vermehrt auf ihre Erinnerungen zurück oder müssen sich Patientendetails in einem weiteren Medium extern aufrufen. Darüber hinaus wird das Entlassdatum in Kliniken meist intern und kurzfristig am selben Tag festgelegt. Änderungen des Entlassdatums erreichen in wenigen Fällen den Hausarzt oder die Apotheke. So besteht Ungewissheit darüber, wann Patienten eintreffen und welche Medikamente vorrätig sein sollten. Vor allem bei Entlassungen kurz vor dem Wochenende führt dies zu erheblichen Herausforderungen. Die Kommunikation zwischen Klinikum, Hausarzt und Apotheke verläuft meist über den Patienten oder dessen Angehörige als Informationsübermittler. Diese werden der Aufgabe häufig nicht gerecht, da Unklarheit über die aktuelle Behandlung oder den weiteren

\footnotetext{
1 Vollständiger Ist-Prozess: https://t1p.de/187i.

2 Vollständiger Soll-Prozess: https://t1p.de/1yyw.
} 


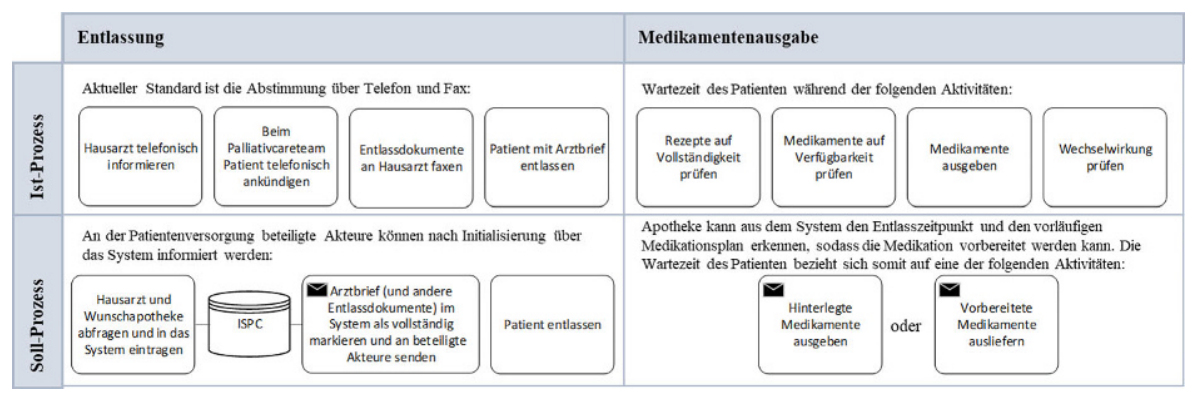

Abb. 2 Prozessschritte im Ist- und Soll-Prozess bei der Entlassung und der Medikamentenabgabe

Verlauf besteht. Laut der Apotheker unserer Fokusgruppe erreichen die Apotheken oftmals unvollständige Rezepte, sodass bspw. die Packungsgröße nicht eindeutig benannt ist. Die Apotheken sind bei der Medikationseinstellung oft nicht eingebunden und können zur bestehenden Medikation nur erschwert Rückfragen stellen, da der Ansprechpartner für Rückfragen häufig nicht eindeutig dargestellt ist oder der zuständige Arzt schwer zu erreichen ist. Das Prüfen von Wechselwirkungen ist bei Palliativpatienten, die in der Regel multimorbid sind, d.h. mehrere Medikamente gleichzeitig einnehmen, von hoher Relevanz. Bei einer Patientenentlassung werden Medikationspläne in Arztpraxen handschriftlich ergänzt, was Leseprobleme hervorrufen kann, oder Pläne werden neu ausgedruckt und dem Patienten zusammen mit dem alten Plan mitgegeben. Daraus folgt oftmals ein hoher Organisationsaufwand für die Instanz (meist der Apotheker oder das Palliativnetz), die den Medikationsplan aus den Entlassdokumenten anschließend erhält, zwischen verschiedenen Dokumenten entscheiden und handschriftliche Ergänzungen lesen muss.

Abb. 2 stellt die Prozessschritte zweier zentraler Prozessbereiche dar: die Entlassung und die Medikamentenausgabe. Herausforderungen vor allem bzgl. der Kommunikation sind der Zeile des Ist-Prozesses zu entnehmen. Diese sind den Aktivitäten im Soll-Prozess gegenübergestellt. So wird deutlich, inwiefern die Prozessschritte digitalisierter und schlanker werden können.

\subsection{Prozessanalyse: Anforderungen an eine sektorenübergreifende Plattform im Gesundheitswesen}

In diesem Anwendungsfall konnte in den vorher geführten Experteninterviews festgestellt werden, dass generell eingesetzte Plattformen im Gesundheitssektor den spezifischen Anforderungen des Palliativbereichs nicht gerecht werden. Als Beispiel kann der Bundeseinheitliche Medikationsplan (BMP) herangezogen werden. Die Inhalte des BMP sind maschinenlesbar in einem Barcode festgehalten, um Inhalte schneller verfügbar zu machen und automatisch in digitale Anwendungen übertragen zu können. Auszuwählende Zeitpunkte des BMP zur Medikationsverabreichung sind nur morgens, mittags oder abends, wobei ein Palliativpatient teilweise alle zwei Stunden ein Medikament einnehmen muss. Darüber hinaus kann aktuell kein etabliertes System als Standard im flächendeckenden Einsatz ausgemacht werden, das den Austausch zwischen den Gesundheitsakteuren fokussiert. Aus diesem 
Grund wurde die Marktrecherche nur innerhalb des Palliativsektors durchgeführt und begrenzt sich daher auf Systeme, die bereits von Palliativnetzen verwendet werden. So kann die bestehende Datenbasis genutzt und Palliativnetze müssen nicht zusätzlich von einem Wechsel überzeugt werden. In der Palliativversorgung konnten mithilfe einer Marktrecherche folgende drei Plattformen identifiziert werden: Informationsystem Palliative Care (ISPC), Pallidoc und Pallida.

Im Umfeld dieses Forschungsvorhabens wird weitestgehend das im Palliativbereich stark verbreitete ISPC eingesetzt - so auch von den zwei in diese Studie involvierten Palliativnetzen. Folglich fiel die Entscheidung im Rahmen dieser Untersuchung darauf, das webbasierte ISPC für eine Weiterentwicklung zu nutzen. Vor allem das bereits eingepflegte Rollen- und Rechtemanagement bietet die Möglichkeit, das Vorhaben sinnvoll und effizient auf Basis einer One-Platform-Solution durchzuführen und kein weiteres System zu entwickeln, das wiederum angebunden werden müsste. Neben dem Kernprodukt für die häusliche Palliativversorgung existiert auch das Derivat ISPC clinic, bei dem durch Schnittstellen zu den Krankenhausinformationssystemen (KIS) existierende Patientenakten und Abschlussberichte importiert sowie exportiert werden können. Assistenten können Arztbriefe anlegen, die durch Ober- und Chefärzte freigegeben werden. Eine Patientenliste ermöglicht den übersichtlichen Zugriff auf die Daten der Palliativpatienten, darunter fällt die Dokumentation von Symptomverläufen, Medikationsplänen und Arztbriefen. Darüber hinaus können Formulare (teil-)automatisiert erstellt werden, indem auf patientenspezifische Daten im System zurückgegriffen wird. Die Systemsicherheit wird durch eine mehrstufige Benutzerauthentifizierung und Verschlüsselung gewährleistet.

Der Ist-Prozess wurde auf Schwachstellen und Probleme geprüft, woraus Verbesserungspotenziale mithilfe einer digitalen Lösung abgeleitet werden konnten. Mithilfe dieser lassen sich Anforderungen an eine Plattform und anschließend der Soll-Prozess ableiten. Die Probleme lagen vor allem im Kommunikations- und Dokumentationsbereich, was aufzeigt, dass es an einer gemeinsamen Austauschplattform zwischen verschiedenen Gesundheitsakteuren mangelt. Das ISPC konnte mittels der identifizierten Plattform-Anforderungen für den Palliativbereich auf die Klinikmitarbeiter-, Apotheken- und Hausarztrolle erweitert werden. Die Anforderungen und teils Erweiterungen an die Plattform sind in Tab. 1 dargestellt und sind den zwei Bereichen Kommunikation (K) und Dokumentation (D) zugeordnet. Mithilfe eines Medikationsplans und eines Dokumentenmanagements kann die Dokumentation nachhaltig gesichert werden, indem die Patientendetails von der Entlassung bis zur täglichen Versorgung sukzessiv ergänzt oder verändert werden. Vor allem ist die Patientenorientierung im Bereich Plattformen im Gesundheitswesen wichtig. Mit einem patientenorientierten System ist gemeint, dass bspw. der Medikationsplan und eingehende Änderungen per Chat immer der Patientenakte angehängt sind und Patientendetails so leicht aufzurufen sind. Ein Chat beschreibt in diesem Fall eine einfache Nachrichtenfunktion, angelehnt an bekannte Kommunikationsapplikationen, um einen einfacheren Austausch zu ermöglichen. Hinsichtlich der Dokumentation kann ein digitaler Medikationsplan mit Anbindung an die ABDA- 
Tab. 1 Plattformanforderungen und Erweiterungen des bereits existierenden ISPC

\begin{tabular}{|c|c|c|}
\hline $\mathrm{K}$ & \multicolumn{2}{|c|}{$\mathrm{D}$} \\
\hline \multicolumn{3}{|c|}{ Einsehen, Einpflegen und Ändern der Patientendaten } \\
\hline- & $\mathrm{x}$ & Import von Stammdaten der Patienten aus dem KIS oder Praxissystem \\
\hline- & $\mathrm{x}$ & Dokumentenablage (für Arztbrief, BMP etc.) \\
\hline- & $\mathrm{x}$ & $\begin{array}{l}\text { Einpflegen und Zuordnen von Gesundheitsakteuren zu dem jeweiligen Pati- } \\
\text { enten (z.B. Stammapotheke) }\end{array}$ \\
\hline $\mathrm{x}$ & $\mathrm{x}$ & $\begin{array}{l}\text { Einsicht auf die Patientendetails und Entlassinformationen sowie -dokumente } \\
\text { nach erfolgter Freigabe }\end{array}$ \\
\hline- & $\mathrm{x}$ & $\begin{array}{l}\text { Rollen- und Rechtemanagement: Anpassung der Ansicht der digitalen Akte } \\
\text { auf Akteurs-relevante Einträge }\end{array}$ \\
\hline- & $\mathrm{x}$ & $\begin{array}{l}\text { Spezifische Verlaufskürzel zur Dokumentation von Eintragungen und Ände- } \\
\text { rungen in der Patientenakte }\end{array}$ \\
\hline \multicolumn{3}{|c|}{ Austausch } \\
\hline $\mathrm{x}$ & $\mathrm{x}$ & $\begin{array}{l}\text { Patientenorientierter, langfristiger Kommunikationskanal bspw. Apotheker- } \\
\text { Rückmeldung zur Medikation }\end{array}$ \\
\hline $\mathrm{x}$ & - & Asynchrone Kommunikation: Chat \\
\hline $\mathrm{x}$ & - & $\begin{array}{l}\text { Kommunikation ist zwischen Gesundheitsakteuren und zwischen Patient und } \\
\text { Gesundheitsakteuren möglich }\end{array}$ \\
\hline $\mathrm{x}$ & $\mathrm{x}$ & Kontaktdaten aller beteiligten Akteure und präferierte Kontaktart \\
\hline $\mathrm{x}$ & - & $\begin{array}{l}\text { Automatische E-Mail-Benachrichtigung von Gesundheitsdienstleistern bei } \\
\text { für diese relevanten Eintragungen }\end{array}$ \\
\hline \multicolumn{3}{|c|}{ Medikationsbezogene Funktionen } \\
\hline $\mathrm{x}$ & $\mathrm{x}$ & $\begin{array}{l}\text { Medikamentenbestellung Standardmaske aus Medikationsplan möglich (vom } \\
\text { Hausarzt zum Apotheker) }\end{array}$ \\
\hline $\mathrm{x}$ & $\mathrm{x}$ & Medikamentenbestellung: Wahl zwischen Botendienst und Abholung \\
\hline $\mathrm{x}$ & $\mathrm{x}$ & Rezeptbestellung Standardmaske (vom Apotheker zum Hausarzt) \\
\hline $\mathrm{x}$ & $\mathrm{x}$ & Rezeptübersendung durch Hausarzt \\
\hline- & $\mathrm{x}$ & $\begin{array}{l}\text { Darstellung und Bearbeitung eines Medikationsplans von dem Patienten } \\
\text { (u. a. Einscannen des BMP) }\end{array}$ \\
\hline- & $\mathrm{x}$ & $\begin{array}{l}\text { Einsehen und Ändern des Medikationsplans von allen an der Patientenversor- } \\
\text { gung beteiligten Akteuren }\end{array}$ \\
\hline- & $\mathrm{x}$ & $\begin{array}{l}\text { Möglichkeit, bei Bedarf, eine Medikationsänderung über das System dem } \\
\text { Hausarzt vorzuschlagen }\end{array}$ \\
\hline
\end{tabular}

Datenbank ${ }^{3}$ und eine digitale Dokumentenablage (für bspw. den Arztbrief) als Single Point of Truth dienen, der durch eine standardisierte Bestellmaske keine Fehler in der Medikationsbestellung zulässt.

\subsection{Prozessverbesserung: Einsatz der Plattform ISPC und Verantwortungsverteilung im Soll-Prozess}

Es wurde ein Soll-Prozess, der die Einbindung einer gemeinsamen Austauschplattform vorsieht, definiert und nach erfolgter Erweiterung der ISPC-Plattform

\footnotetext{
3 ABDA steht für Bundesvereinigung Deutscher Apothekerverbände e. V.: Die ABDA-Datenbank enthält umfassende Informationen zu Fertigarzneimitteln und deren Inhaltsstoffen (siehe https://abdata.de/ datenangebot/abda-datenbank/).
} 
spezifiziert ${ }^{2}$. Dieser Soll-Prozess muss nicht in der Gesamtheit, sondern kann auch in Teilen umgesetzt werden. Die leichteste Umsetzung besteht in der Zusammenarbeit zwischen Palliativnetz und einer Partnerapotheke, was aufgrund der nächtlichen schnellen Verfügbarkeit von Betäubungsmitteln einen hohen Nutzen bietet. Auch von Vorteil ist die Zusammenarbeit nur zwischen Palliativnetz und Palliativstation einer Klinik hinsichtlich des Übergangs von stationärer zu ambulanter Versorgung. Je nach Versorgungsstruktur, ob ein Hausarzt auch Palliativarzt ist oder eine Klinik im Nachtdienst die ambulante Palliativversorgung übernimmt, gestaltet sich die Einbindung des ISPC entweder einfacher oder umfangreicher. Im Folgenden wird der vollständige Soll-Prozess beschrieben, der alle Gesundheitsakteure berïcksichtigt: Klinik, Hausarzt, Apotheke sowie Palliativnetz.

\subsubsection{Soll-Prozess für die Klinik}

Der Palliativsektor in der Klinik kann als klinisches System das ISPC bereits nutzen, sodass das Palliativnetz und die Palliativstation in einem System arbeiten und der Übergang von stationär in ambulant oder vice versa keinen Aufwand bedeutet. Sobald feststeht, dass ein Palliativpatient entlassfähig ist, und ein anderes KIS als das ISPC genutzt wird, ist dieser Patient mit seinen Stammdaten von einem Klinikmitarbeiter im ISPC digital anzulegen. Die Daten können automatisiert übertragen werden, wenn eine Schnittstelle vom ISPC zum KIS besteht. Die Stammdaten des Patienten umfassen neben dem Namen auch die Adresse, den zuständigen Hausarzt und die Wunsch-Apotheke des Patienten mit ISPC-Anbindung. Dafür wird eine Einwilligung für die Datenweitergabe der Entlasspapiere an die beteiligten Gesundheitsakteure vom Patienten eingeholt. Neben den Stammdaten des Patienten können die Klinikmitarbeiter das Entlassdatum eintragen. So können die zuständigen Ärzte und Apotheker einen einheitlichen, aktuellen Stand über die Entlassung des Palliativpatienten erhalten. Die Entlassdokumente können im System bereitgestellt werden, wodurch der Hausarzt per Email über einen neuen Eintrag im ISPC informiert wird. Die Entlassdokumente müssen nicht postalisch oder per Fax geschickt werden, wodurch Bürokratie reduziert sowie eine zentrale, digitale und patientenzentrierte Plattform für verschiedene Gesundheitsakteure geschaffen wird. Auch die Klinik profitiert von der Prozessumstellung, da Patientenakten nicht mehr gedruckt, sondern diese digital übermittelt sowie abgelegt werden können und Rückfragen schneller zuzuordnen sind. Die Einschreibung in das Palliativnetz, welche dem Hausarzt gewährt bleibt, ist nicht gleichzusetzen mit dem Anlegen eines Patienten im ISPC. Somit hat nur der zuständige Hausarzt die Berechtigung, nach erfolgter Einschreibung des Patienten in das Palliativnetz den Marker im ISPC zu setzen.

\subsubsection{Soll-Prozess für den Hausarzt}

Der Hausarzt erhält per Mail eine Benachrichtigung, dass sich etwas im ISPC verändert hat. Dort kann er die Unterlagen von der Klinik einsehen und die Medikation anpassen. Sofern der Medikationsplan noch nicht vorhanden ist, kann er dafür den Barcode auf dem BMP per Handykamera scannen und automatisch einspielen. Falls der BMP in das System mit den anderen Entlassdokumenten hochgeladen wurde, 
kann der Barcode auf dem PDF direkt im ISPC gescannt werden. Eine Detaillierung oder Änderung der Medikation, wie z.B. zusätzliche Einnahmezeitpunkte, können hier in einem einheitlichen Format vorgenommen werden. Bei der Eintragung sind dem Palliativnetz diese Dokumente nicht mehr über Fax zuzusenden, sondern können bei Bedarf auf der Plattform patientenzentriert abgerufen werden. Der Hausarzt hat die Möglichkeit, den Palliativpatienten über das ISPC in das Palliativnetz einzuschreiben und anzumelden. Auch alle für die Abrechnung relevanten Daten werden im ISPC dokumentiert, sodass diese strukturiert exportiert (per BDT) und in ein für die Krankenversicherung zertifiziertes System übergeben werden können.

Während des Hausarztbesuches kann der Patient entscheiden, ob er die Medikamente in der zuständigen Apotheke abholen oder nach Hause geliefert bekommen möchte. Der Palliativarzt kann ebenfalls beim Hausbesuch eine Medikamentenbestellung über das ISPC abschicken. Eine standardisierte Medikamentenbestellung kann direkt aus dem ISPC per Knopfdruck abgewickelt werden, damit die vom Patienten gewünschte Apotheke mit ISPC-Anbindung alle notwendigen Informationen im Vorhinein erhält und die Bestellung vorbereiten kann. Das Rezept wird nach wie vor ausgedruckt und dem Patienten übergeben.

\subsubsection{Soll-Prozess für das Palliativnetz}

Das Palliativnetz betreut den Patienten ambulant außerhalb der normalen Sprechzeiten des Hausarztes, weshalb eine engmaschige Zusammenarbeit und gemeinsame Datenbasis dieser beiden Akteure sinnvoll ist. Für den PKD entfällt durch die digitale Freigabe des Patienten inklusive des Medikationsplans die fehleranfällige Übertragung der handschriftlichen Ergänzungen des Hausarztes. Eigene Vorschläge für Änderungen der Medikation kann der PKD nach Rücksprache mit dem Hausarzt umsetzen. Der PKD-Arzt kann per Smartphone oder Tablet jederzeit mobil die Stammdaten und Medikation des Patienten einsehen. Bei Bedarf eines neuen Medikaments kann der Palliativarzt eine Medikamentenbestellung per Standardfunktion in ISPC abschicken, ein Rezept ausstellen und je nach Patientenwunsch zur Abholung oder Zustellung per Botendienst bestellen. Die Wunsch-Apotheke mit ISPCAnbindung kann im System ausgewählt werden und die Bestellung mit mobilem Internet abgewickelt werden. So kann eine vermehrt vorkommende, zeitkritische Versorgung von Palliativpatienten durch eine standardisierte Medikamentenbereitstellung bei einer Apotheke vor Ort mit ISPC-Anbindung gewährleistet werden. Besteht keine dringende Versorgungsnot oder keine Internetverbindung kann der PKD nach seinen Patientenbesuchen die Apothekenbestellungen konsolidiert aufgeben.

\subsubsection{Soll-Prozess für die Apotheke}

Eine Apotheke mit ISPC-Anbindung wird per E-Mail benachrichtigt, sobald eine Bestellung vom Hausarzt oder vom Palliativnetz eingegangen ist. Im ISPC kann der Apotheker den vollständigen Medikationsplan auf etwaige Wechselwirkungen prüfen. Sofern der Apotheker Unstimmigkeiten entdeckt, können die Kontaktdaten des zuständigen Arztes abgerufen werden oder die Kommunikation kann über das Nachrichtensystem von ISPC mit Verlinkung auf den Patienten asynchron erfolgen. Eine 
eingegangene Bestellung wird zur Abholung vorbereitet oder, wenn eine Lieferung gewünscht wurde, zum nächsten Zeitpunkt von einem pharmazeutischen Boten zum Patienten ausgeliefert werden. Dabei nimmt dieser Bote das Rezept für die Medikamente an der Haustür des Patienten entgegen. Da regelmäßig die Anforderung einer zeitkritischen Versorgung von Medikamenten oder Betäubungsmitteln für Palliativpatienten außerhalb der Öffnungszeiten vorkommt, kann nur eine Apotheke vor Ort mit der bestehenden Infrastruktur diesen Dienst zu jeder Zeit sicherstellen.

\subsection{Prozessverbreitung und -implementierung}

Der Einsatz von digitalen Technologien intensiviert die Betreuung des Palliativpatienten, kann die Versorgungssicherheit sowie -qualität verbessern und der Überlastung im Gesundheitswesen nachhaltig entgegenwirken (Schnell et al. 2019). Nach der Erweiterung des ISPC wurden die Nützlichkeit und Notwendigkeit des resultierenden Soll-Prozesses abgefragt. Apotheker waren motiviert, sich in neue Systeme einzuarbeiten sowie den Soll-Prozess zu übernehmen und auszuprobieren. Klinikmitarbeiter und Palliativärzte waren zum Teil interessiert, dann jedoch nicht bereit, tiefgehende Veränderungen ihrer Prozesse einzugehen. Nach Angaben der Palliativärzte ist der Grund für die Ablehnung neuer Prozesse die Überlastung des Gesundheitssystems und dadurch der Mangel an Ressourcen und Zeit, alle Beteiligten auf einen neuen Prozess zu schulen. Auch partielle Veränderungen wurden abgelehnt, teilweise aufgrund des zu Beginn anfallenden Mehraufwands. Bei den Hausärzten lag darüber hinaus die Schwierigkeit darin, grundsätzlich Kontakt zu diesen herzustellen. Aus Umstrukturierungen der Arbeitsabläufe und fehlendem Know-How resultiert häufig eine Zurückhaltung gegenüber einem neuen System (Yogesh und Gaurav 2019). In einem nächsten Ansatz können unterschiedliche Methoden angewendet werden, die Akzeptanz neuer Technologien zu fördern. Eine Argumentenbilanz kann als Hilfsinstruments dienen, sodass die Stakeholder ihre Vor- und Nachteile auf einem Blick sehen und sich für oder gegen die Einführung einer be-

\begin{tabular}{|c|c|c|c|c|}
\hline Patient (und Angehörige) & Palliativnetz & Apotheke & Hausarzt & Klinik \\
\hline Höhere Versorgungsqualitāt & $\begin{array}{l}\text { Automatisches Einlesen des } \\
\text { Medikationsplans }\end{array}$ & \multicolumn{3}{|c|}{$\begin{array}{l}\text { Mitbestimmung der Kommunikationsart: } \\
\text { Anfrage kann per Chat oder telefonisch zu einem selbst gewählten Zeitpunkt erfolgen }\end{array}$} \\
\hline $\begin{array}{l}\text { Weniger organisatorischen } \\
\text { Aufwand (Dokumente) }\end{array}$ & $\begin{array}{l}\text { Mobile Übersicht des } \\
\text { Medikationsplans }\end{array}$ & $\begin{array}{l}\text { "Vier-Augen-Prinzip“ über } \\
\text { die Medikation }\end{array}$ & $\begin{array}{l}\text { „Vier-Augen-Prinzip“ über } \\
\text { die Medikation }\end{array}$ & $\begin{array}{l}\text { Telefonische Abstimmung } \\
\text { mit Palliativnetz und }\end{array}$ \\
\hline $\begin{array}{l}\text { Nicht länger der Übermittler } \\
\text { relevanter Informationen }\end{array}$ & $\begin{array}{l}\text { Medikationsänderungen } \\
\text { möglich (erfahrungsbasiert) }\end{array}$ & $\begin{array}{l}\text { Höhere Arzneimittel- } \\
\text { therapiesicherheit }\end{array}$ & $\begin{array}{l}\text { Monitoring über das } \\
\text { Patientenwohl }\end{array}$ & $\begin{array}{l}\text { Hausarzt nicht notwendig: } \\
\text { Eintragung über das System }\end{array}$ \\
\hline $\begin{array}{l}\text { Wegersparnis (optional } \\
\text { Apothekenbotendienst) }\end{array}$ & \multirow{2}{*}{$\begin{array}{l}\text { Medikamentenbestellung und } \\
\text { Rezeptübersendung über das } \\
\text { System möglich }\end{array}$} & \multicolumn{2}{|c|}{$\begin{array}{l}\text { Medikamentenbestellung und Rezeptübersendung über das } \\
\text { System möglich }\end{array}$} & $\begin{array}{l}\text { Keine analoge Dokumenten- } \\
\text { ablage mehr notwendig }\end{array}$ \\
\hline & & $\begin{array}{l}\text { Standardisierte Bestellung } \\
\text { (weniger fehleranfällig) }\end{array}$ & Erleichterte Abrechnung & $\begin{array}{c}\text { Sicherstellung des } \\
\text { richtigen Empfängers }\end{array}$ \\
\hline & \multicolumn{3}{|c|}{ Entlasszeitpunkt einsehbar, wodurch erhöhte Planbarkeit gegeben ist } & \\
\hline & \multicolumn{4}{|c|}{ Nahtloser, sektorenübergreifender Austausch: datenschutz-bedenkliches Fax nicht mehr notwendig } \\
\hline & \multicolumn{4}{|c|}{ Single Point of Truth: Eine einheitliche Datenbasis für alle Akteure (Missverständnisse bleiben aus) } \\
\hline & \multicolumn{4}{|c|}{$\begin{array}{l}\text { Asynchrone Kommunikation über den gesicherten Chat, wodurch Ressourcen eingespart werden können } \\
\text { (Erreichbarkeit gewährleistet, richtiger Ansprechpartner, Abarbeiten der Anfrage je nach Auslastung) }\end{array}$} \\
\hline \multirow{2}{*}{$\begin{array}{l}\text { Legende } \\
\square \text { Vorteile } \\
\text { Nachteile }\end{array}$} & $\begin{array}{l}\text { Initiale Einarbeitung des } \\
\text { Medikationsplans }\end{array}$ & \multicolumn{3}{|c|}{ Einführung des Systems, Schulung der Mitarbeiter, Veränderung des Arbeitsprozesses } \\
\hline & $\begin{array}{l}\text { Zusătzliches mobiles Gerăt } \\
\text { bei/vor/nach der Behandlung }\end{array}$ & & & $\begin{array}{l}\text { Erhöhter Aufwand: } \\
\text { Initialisierung in das System }\end{array}$ \\
\hline
\end{tabular}

Abb. 3 Argumentenbilanz zu intersektoralen Plattformen im Gesundheitswesen 
reichsübergreifenden Plattform entscheiden können (Witte 2020). Abb. 3 zeigt eine Argumentenbilanz für den in diesem Vorhaben vorgestellten Anwendungsfall.

\section{Diskussion und Implikationen}

Der im Rahmen dieser Untersuchung vorgestellte Ansatz zeigt am Beispiel des Palliativsektors, welche Anforderungen an Plattformen bestehen, die auf eine intersektorale Kommunikation im Gesundheitswesen mit dem Fokus Medikationsmanagement abzielen. Darüber hinaus wird dargestellt, wie die Verantwortlichkeiten verschiedener Aufgaben zwischen den Gesundheitsakteuren aufgeteilt werden könnten. Plattformen sind im Gesundheitswesen und zwischen unterschiedlichen Gesundheitseinrichtungen relevant, da die aktuell zum Großteil eingesetzte redundante, analoge Dokumentenablage zu Kommunikationsproblemen und Mehraufwand führt. Neben dem administrativen Aufwand können in der Folge auch medizinische Probleme auftreten. Arzneimittelbezogene Probleme verursachen vermeidbares Patientenleid (Reimers und Klein 2015). Ein Hindernis eines digitalen Austauschs ist oftmals nicht die Reife der Technologien, sondern die Akzeptanz und Motivation aller beteiligten Gesundheitsakteure, ihre Prozesse umzustellen und von der gewohnten Prozedur abzulassen - analog zu den Herausforderungen in der Prozessoptimierung (Sawatzky et al. 2018). Das Einführen eines regelmäßigen Prozessmanagements-Lebenszyklus, wie in diesem Vorhaben vorgestellt, und damit einer Prozessumstrukturierung und Plattformnutzung kann langjährig bestehende, analoge Prozesse im Gesundheitswesen systematisch (teil)digitalisieren und damit verschlanken. Anhand der elektronischen Fallakte wird aufgezeigt, dass eine flächendeckende Vernetzung funktionieren kann, jedoch aktuell aufgrund der teilweise fehlenden Praktikabilität noch nicht genutzt wird. Eine zentrale, einheitliche Lösung für u.a. eRezept und eMedikationsplan sind in Planung und werden in der Zukunft entwickelt ${ }^{4}$. Für den Übergang bis diese einheitliche, standardisierte Lösung geschaffen ist, existieren jedoch bereits Plattformlösungen wie das ISPC, die funktionieren und für eine Zusammenarbeit im Gesundheitssektor genutzt werden können und sollten.

Anhand des aufgenommenen Prozesses ist deutlich zu erkennen, dass Medikationsversorgung und -management ein Schlüsselelement in der Gesundheitsversorgung ist und ein Bedarf an einem intersektoralen Medikationsmanagement generell im Gesundheitswesen besteht. Auch mit kleinen Veränderungen kann die Gesundheitsversorgung diesbezüglich verbessert werden, indem bspw. alle Kliniken den BMP ausstellen. Dies ist vorteilhaft für die nachfolgenden Gesundheitsdienstleister, da der BMP einen Barcode zum digitalen Ablesen der darauf enthaltenden Informationen umfasst. Seit Oktober 2016 haben Patienten einen gesetzlichen Anspruch auf diesen BMP, wenn sie mindestens drei verschreibungspflichtige Medikamente dauerhaft einnehmen oder anwenden ${ }^{5}$. Obwohl Kliniken somit verpflichtet sind, im Rahmen der Entlassung einen BMP zur Verfügung zu stellen, ist dieser im unter-

\footnotetext{
4 Bspw. gematik: siehe https://www.gematik.de/anwendungen/, abgerufen am: 05.05.2021.

5 KBV 2016: Vereinbarung eines bundeseinheitlichen Medikationsplans, siehe https://www.kbv.de/media/ sp/Medikationsplan.pdf, abgerufen am: 10.07.2020.
} 
suchten Bereich nur von einer von sechs Kliniken bisher eingeführt worden. Strebt eine Klinik eine starke Einbindung ihrer Palliativstation in den gesamten Versorgungsprozess an, kann diese das ISPC statt ein KIS für Stammdatenmanagement und Medikationsplan nutzen, um generell die Versorgungswechsel von stationär zu ambulant zu vereinfachen. Im Gegensatz zum Fax ist bei der Anwendung von Plattformen der richtige Empfangspartner sichergestellt. Hausärzte wie auch die Klinik werden von Vorteilen profitieren, dass eine zentrale, digitale Dokumentenablage und Medikationsabstimmung die Prozesse und Abstimmungen vereinfachen wird. Dies zeigen zahlreiche Beispiele, in denen das ISPC bereits im Einsatz ist wie bspw. Klinikum der Universität München, Uniklinikum Göttingen und Universitätsklinikum Würzburg. Der Apotheker war offen gegenüber einer Einarbeitung in Fremdsysteme für eine verbesserte und vernetzte Versorgung, vor allem bzgl. Akutsituationen. Aktuell arbeiten Palliativnetze meist wegen der zeitkritischen Medikamentenversorgung mit einer Partnerapotheke über das ISPC. Bei der Bereitschaft von mehreren Apotheken für den Notdienst könnte dies ausgeweitet werden auf die nächstgelegene oder Wunsch-Apotheke des Patienten.

Aufgrund der Nähe und der Expertise zu Arznei und Patienten sowie der Verbindung zu allen Gesundheitsakteuren könnte der Apotheker zukünftig im Zentrum der Kommunikation stehen. Die Rolle des Apothekers kann an Bedeutung gewinnen, indem er die Rolle eines Gesundheitslotsen einnimmt und die Kommunikation zwischen Patienten und den anderen Gesundheitsakteuren koordiniert. Dieser scheint prädestiniert dafür zu sein, da er dem Patienten bereits eine niedrigschwellige Anlaufstation mit viel Empathie bietet und deutlich mehr Kunden- bzw. Patientenkontakte als ein Arzt hat (EPatient Survey 2020). Ein Apotheker vor Ort kennt die Verschreibungsgewohnheiten der Ärzte vor Ort. Auch das hilft Fehler zu vermeiden. Auch kurze elektronische Kommunikation zwischen den Heilberuflern außerhalb normaler Dienstzeiten und schnelle Ausführung seitens der Apotheke dienen dem Wohle des Patienten. Die Veränderung der Apothekerrolle Richtung Gesundheitslotse bzw. Gesundheitskoordinator könnte möglicherweise die Überlastung im Gesundheitswesen reduzieren. Forschungsprojekte im Gesundheitsbereich sollten diesen Aspekt somit fokussieren und die Apotheke als Gesundheitskoordinator in der Praxis und die damit zusammenhängenden Geschäftsmodelle weiter erforschen. Des Weiteres könnte eine Erweiterung des BMP mit einem einheitlichen Format für spezifische Einnahmezeitpunkte für eine Verschlankung des Prozesses sorgen.

Ein weiterer Aspekt ist, dass bereits viele Plattformen und damit existierendes Know-How im Markt besteht. Da eine bestehende Plattform bereits Nutzergruppen aufweisen, könnte es in Hinblick auf die Akzeptanz einfacher sein, eine Weiterentwicklung statt einer Neuentwicklung als Standard zu etablieren. Im Rahmen von Forschungsprojekten wird bei Neuentwicklungen von Systemen die Marktreife teilweise nicht erreicht und nach Ablauf der Forschungsprojektlaufzeit ist die Nachhaltigkeit aufgrund des fehlenden Betreibers oft nicht gesichert. In dem vorgestellten Fall ist es effizienter und zielführender auf marktreife Lösungen zurückzugreifen, da die Prozessumgestaltung im Vordergrund steht. In Zukunft könnte überlegt werden, jungen Unternehmen und evaluierenden Forschungsinstitutionen eine Weiterentwicklung und wissenschaftliche Evaluierung bereits eingesetzter Plattformen als zentrales Ergebnis eines Forschungsprojekts zu ermöglichen. Plattformhersteller sollten bei der 
Entwicklung oder Erweiterung ihrer Systeme weitere Gesundheitsakteure oder verwandte Patientengruppen (im Sinne eines User Centred Designs) einbeziehen, sodass Synergieeffekte entstehen und größere Nutzergruppen angesprochen werden können. In Bezug auf diesen Anwendungsfall, wäre es bspw. möglich, den Apotheker als Gesundheitskoordinator zu betrachten oder den Patienten und die Angehörigen in die Plattform für eine erleichterte Kommunikation mit dem Palliativ-Pflegeteam und für ein digitales Selbstmanagement wie bspw. Schmerzdokumentation zu integrieren (Reis et al. 2013; Saldaña et al. 2015).

\section{Fazit}

Diese Untersuchung zeigt anhand des Palliativsektors, dass die intersektorale Kommunikation und einheitliche Dokumentation im Gesundheitswesen noch nicht etabliert ist, aber dafür bereits Plattformlösungen bestehen, die genutzt werden können. Anhand der abgeleiteten Anforderungen konnte das ISPC mittels Rechtemanagement auf die Klinikmitarbeiter-, Apotheken- und Hausarztrolle erweitert und die Freigabe auf die entsprechenden Informationen und Funktionen vergeben werden. In Hinblick auf die Forschungsfrage wird dargelegt, wie die Kommunikation und Dokumentation im Gesundheitswesen mithilfe der Erweiterung bestehender Plattformen integriert und gefördert werden können. Eines der größten Hindernisse ist dabei die Motivation der Akteure, die Plattform einzuführen bzw. die eigenen langjährigen Prozesse zu verändern. Dabei kann das Verständnis helfen, dass bereits kleinere Veränderung - wie bspw. die Einführung des BMP in Kliniken oder der Austausch von Palliativnetz und einer Partnerapotheke über das ISPC - die Zusammenarbeit und damit Versorgungsstrukturen deutlich erleichtern und verbessern können.

Der Artikel stellt die Vorteile des Einsatzes einer bereichsübergreifenden Plattform im Gesundheitswesen dar, um Transparenz für den Mehrwert einer Austauschplattform und eines Single Point of Truth zu schaffen, sodass Probleme bzgl. der Datenaktualität oder Erreichbarkeit der Vergangenheit angehören können. In diesem Prozess wird deutlich, wie wichtig die Apotheke vor Ort mit der bestehenden Infrastruktur in der aktuellen, zum Teil zeitkritischen Gesundheitsversorgung sind. Der Ansatz, bestehende Plattformen zu erweitern, wurde verfolgt, um einerseits die vorhandene IT-Infrastruktur am Markt zu nutzen und andererseits die Akzep$\operatorname{tanz} \mathrm{zu}$ erhöhen, da Nutzer durch bestehende Anwendergruppen voraussichtlich leichter für eine Nutzung überzeugt werden können. Zentrale Anforderungen an bestehende Plattformen können wie folgt zusammengefasst werden: Plattformen zur kollaborativen Arbeit sollten ein Rollenberechtigungskonzept, eine jeweils auf die Gesundheitsfunktion angepasste Sichtmaske, ein Nachrichtensystem zwischen den Gesundheitsakteuren und ergänzende Funktionen, die durch die gemeinsame Nutzung ermöglicht werden (in diesem Fall Medikationsmanagement), erfüllen.

Als Limitation dieses Vorhabens ist zu nennen, dass der abstrahierte Ist- und Soll-Prozess mit Gesundheitsakteuren von nur zwei verschiedenen Palliativnetzen modelliert und verifiziert worden ist. In anderen Regionen kann sich die Form der Palliativversorgung von diesen unterscheiden. Für die Zukunft soll dieser Artikel Gesundheitsdienstleister und Plattformhersteller motivieren, mittels erweiterter Platt- 
formen die Vernetzung im Gesundheitswesen zu fördern. Im Anschluss an dieses Vorhaben gilt es, zu untersuchen, wie Akteure im Gesundheitswesen verstärkt überzeugt werden können, bestehende Prozesse zu restrukturieren und Plattformen bzw. digitale Lösungen zu nutzen.

Förderung Diese Publikation ist im Rahmen des Forschungsprojekts Apotheke 2.0 (Bundesprogramm Ländliche Entwicklung, Bekanntmachung „Land.Digital“, BMEL/ PT BLE, FKZ 2818LD021) entstanden.

Funding Open Access funding enabled and organized by Projekt DEAL.

Open Access Dieser Artikel wird unter der Creative Commons Namensnennung 4.0 International Lizenz veröffentlicht, welche die Nutzung, Vervielfältigung, Bearbeitung, Verbreitung und Wiedergabe in jeglichem Medium und Format erlaubt, sofern Sie den/die ursprünglichen Autor(en) und die Quelle ordnungsgemäß nennen, einen Link zur Creative Commons Lizenz beifügen und angeben, ob Änderungen vorgenommen wurden.

Die in diesem Artikel enthaltenen Bilder und sonstiges Drittmaterial unterliegen ebenfalls der genannten Creative Commons Lizenz, sofern sich aus der Abbildungslegende nichts anderes ergibt. Sofern das betreffende Material nicht unter der genannten Creative Commons Lizenz steht und die betreffende Handlung nicht nach gesetzlichen Vorschriften erlaubt ist, ist für die oben aufgeführten Weiterverwendungen des Materials die Einwilligung des jeweiligen Rechteinhabers einzuholen.

Weitere Details zur Lizenz entnehmen Sie bitte der Lizenzinformation auf http://creativecommons.org/ licenses/by/4.0/deed.de.

\section{Literatur}

Bönisch S (2016) Was bringt Vernetzung im Gesundheitswesen? Eine wirkungsorientierte Betrachtung interorganisationaler Netzwerke. Springer, Wiesbaden

Coskun S, Basligil H, Baracli H (2008) A weakness determination and analysis model for business process improvement. Bus Process Manag J 14(2):243-261

Dumas M, La Rosa M, Mendling J, Reijers HA (2013) Fundamentals of business process management. Springer, Berlin, Heidelberg, New York

EPatient Survey (2020) Pressemitteilung zum 9. EPatient Survey 2020 der EPatient Analytics GmbH. https://www.epatient-analytics.com/aktuelles/eps2020. Zugegriffen: 10. Juli 2020

Gläser J, Laudel G (2010) Experteninterviews Und Qualitative Inhaltsanalyse. Springer, Berlin, Heidelberg, New York

Mingers J (2001) Combining IS research methods: towards a pluralist methodology. Inf Syst Res 12(3):240-259

Morgan DL (1997) Focus groups as qualitative research, 16. SAGE, Thousand Oaks

Mouttham A, Kuziemsky C, Langayan D, Peyton L, Pereira J (2012) Interoperable support for collaborative, mobile, and accessible health care. Inf Syst Front 14(1):73-85

Mumm JN, Rodler S, Mumm ML, Bauer RM, Stief CG (2021) Digitale Innovation in der Medizin - die COVID-19-Pandemie als Akzelerator von ,digital health“. J Urol Urogynäkol AT 28:1-5

Osmani V, Balasubramaniam S, Botvich D (2008) Human activity recognition in pervasive health-care: supporting efficient remote collaboration. J Netw Comput Appl 31(4):628-655

Ostherr K, Killoran P, Shegog R, Bruera E (2016) Death in the digital age: a systematic review of information and communication technologies in end-of-life care. J Palliat Med 19(4):408-420

Reimers K, Klein S (2015) Arzneimitteltherapiesicherheit im Spannungsfeld von vollständiger Medikationsübersicht, mündigem Patienten und individualisierter Medikation, 3. Aufl. Cuvillier, Göttingen

Reis A, Araújo Pedrosa A, Dourado M, Reis C (2013) Information and communication technologies in long-term and palliative care. Proc Technol 9:1303-1312

Saldaña JJ, Mendoza L, Pitti E, Lombardo MV (2015) Ubiquitous and ambient assisted living ehealth platforms for the republic of Panama: two cases of study. In: Ambient intelligence for health. Springer, Cham, S 138-147 
Sawatzky R, Laforest E, Schick-Makaroff K, Stajduhar K, Reimer-Kirkham S, Krawczyk M, Cohen SR (2018) Design and introduction of a quality of life assessment and practice support system: perspectives from palliative care settings. J Patient Rep Outcomes 2(1):36

Schnell MW, Hochmuth A, Schulz-Quach C (2019) Palliative Care im Zeitalter der Digitalisierung. In: Basiswissen Palliativmedizin. Springer, Berlin, Heidelberg, New York, S 297-302

Witte F (2020) Planungsrisiken und Unvorhersehbares. In Strategie, Planung und Organisation von Testprozessen. Springer Vieweg, Wiesbaden, S 193-203

Wohed P, van der Aalst WM, Dumas M, ter Hofstede AH, Russell N (2006). On the suitability of BPMN for business process modelling. In International conference on business process management (S. 161-176). Springer, Berlin, Heidelberg

Yogesh P, Gaurav S (2019) White paper: Telehealth: breaking down adoption barriers. Infosys, Bengaluru. https://www.infosys.com/industries/healthcare/insights/Documents/breaking-down-adoptionbarriers.pdf. Zugegriffen: 12.11.2020 